\title{
LISTA FAUNÍSTICA DE LOS QUIRONÓMIDOS (DIPTERA, CHIRONOMIDAE) DE MADRID (ESPAÑA) ${ }^{1}$
}

\author{
O. Soriano* y F. Cobo**
}

\begin{abstract}
RESUMEN
En el presente trabajo se aporta por primera vez una lista faunística de los quironómidos (Diptera, Chironomidae) citados en Madrid. También se añaden citas inéditas, siendo seis de ellas nuevas citas para la península Ibérica. En total se recogen 218 especies, tanto de ambientes lóticos como leníticos.

Palabras clave: Diptera, Chironomidae, faunística, Madrid, España.
\end{abstract}

\section{ABSTRACT \\ Check list of the Chironomids (Diptera, Chironomidae) occurring in Madrid (Spain)}

A check list of the Chironomids (Diptera, Chironomidae) recorded from Madrid is provided for the first time. New unpublished records are included, of which six are new for the Iberian Peninsula. 218 species were found, coming from both lotic and lenitic environments.

Key words: Diptera, Chironomidae, faunistic, Madrid, Spain.

\section{Introducción}

Este listado tiene como objetivo presentar de forma sintética toda la información faunística disponible al finalizar 2005 sobre los Quironómidos (Diptera, Chironomidae) presentes en la Comunidad de Madrid. Para ello, además de efectuar una búsqueda exhaustiva del material bibliográfico existente, hemos incorporado información todavía inédita, procedente toda ella del estudio de nuestras propias colecciones, lo cual sirve para completar estudios precedentes que trataban únicamente de la fauna de quironómidos en los ecosistemas lóticos (Soriano,
2001) o de muestreos o citas esporádicas realizadas por autores anteriores (Arias, 1912a y b; Strobl; 1900, 1905; Czerny \& Strobl, 1909; Goetghebuer, 1931; Wülker, 1957; Hirvenoja, 1973; Prat, 1980; Contreras-Lichtenberg, 1986; Cobo et al.,1987; Soriano et al., 1993; Langton, 1994; Michailova et al., 1994), para ello incluimos los resultados de muestreos de diferentes charcas y lagunas que han sido prospectadas en los últimos años, y que creemos conferirá a este trabajo un interés adicional.

La estructura de la lista incluye el nombre específico actualizado nomenclaturalmente según Soriano et al. (1997), las localidades de captura

* Museo Nacional de Ciencias naturales (C.S.I.C.). C/ José Gutiérrez Abascal, 2. 28006-Madrid, España.

** Departamento de Bioloxía Animal, Facultade de Bioloxía, Universidad de Santiago de Compostela. 15782-Santiago de Compostela, España.

Trabajo subvencionado por el Proyecto GR/AMB/0750/2004 de la Comunidad de Madrid. 
(con sus coordenadas U.T.M. ajustadas al máximo posible en el caso de que se pudieran precisar) y, en algunos casos, observaciones sobre la nomenclatura o dudas que pudiera presentar la cita.

La mayoría de las citas se han realizado por determinación de exuvias pupales, es frecuente encontrar por tanto especies que se denominan usando el género seguido de "Pe" y un número. Esta terminología es la utilizada por Langton $(1984,1991)$ para nombrar exuvias que aún no han sido adscritas a una especie determinada o que pudieran pertenecer a ejemplares de especies aún no descritas.

\section{Resultados}

En el trabajo se citan 218 especies para la Comunidad de Madrid, de ellas 6 son citas nuevas para la península Ibérica e islas Baleares. Con anterioridad a esta publicación se registraban para el ámbito Iberobalear 481 especies (Cobo \& Soriano, 2002), por ello ahora el número de especies citadas, se eleva 486.

En la lista que sigue se muestran todas las especies encontradas hasta el momento en la Comunidad de Madrid, y subrayadas las seis especies que son nueva cita para la península Ibérica

\section{Subfamilia TANYPODINAEKieffer, 1906 \\ Tribu TANYPODINIKieffer, 1906 \\ Género TANYPUS Meigen, 1803}

\section{Tanypus kraatzi (Kieffer, 1912)}

LOCALIDADES: Río Guadarrama, 30TVK198688 (Soriano et al, 1993; Soriano, 2001). Laguna del Retamar (Las Rozas de Madrid) 30TVK205855

\section{Tanypus punctipennis Meigen, 1818}

LOCALIDADES: Río Jarama, 30TVL602214 (Soriano, 2001).

Tribu PROCLADIINI Roback, 1971 Género PROCLADIUS Skuse, 1889

Procladius (Holotanypus) choreus (Meigen, 1804)

LOCALIDADES: El Escorial, Madrid (Czerny \& Strobl, 1909; Arias, 1912; Soriano, 2001). Arroyo de Canencia, 30TVL348268; Alberche, 30TUK973632; Río Jarama, 30TVL602214; Río Jarama, 30TVL565139 (Soriano, 2001); Laguna del Garzo (Las Rozas de Madrid), 30TVK266889.

Procladius (Holotanypus) sagittalis (Kieffer, 1909)

LOCALIDADES: Río Lozoya, 30TVL292298 (Soriano et al., 1993; Soriano, 2001)
Procladius (Holotanypus) Pe 1 in Langton, 1991

LoCALIDADES: Río Lozoya, 30TVL292298 (Soriano, 2001). Lagunas de las Ceudas (Las Rozas de Madrid), 30TVK262871.

Procladius (Holotanypus) Pe 3 in Langton, 1991

LOCALIDADES: Río Guadarrama, 30TVK168954 (Soriano, 2001). Lagunas de las Ceudas (Las Rozas de Madrid), 30TVK262871.

Procladius (Holotanypus) Pe 4 in Langton, 1991

LOCALIDADES: San Martín de la Vega, 30TVK5152

Tribu MACROPELOPIINI Fittkau, 1962

Género MACROPELOPIA Thienemann, 1916

Macropelopia nebulosa (Meigen, 1804)

LOCALIDADES: El Escorial, Madrid (Czerny \& Strobl, 1909; Arias, 1912; Cobo et al., 1987; Soriano, 2001). Río Lozoya, 30TVL292298 (Soriano, 2001). Lagunas de la Cervera (Las Rozas de Madrid), 30TVK223846.

Género PSECTROTANYPUS Kieffer, 1909

Psectrotanypus (Psectrotanypus) varius (Fabricius, 1787)

LOCALIDADES: Madrid (Cobo et al., 1987). Río Guadarrama, 30TVK168954 (Soriano, 2001). San Fernando de Henares, 30TVK5476.

Tribu PENTANEURINI Fittkau, 1962 Género ABLABESMYIA Johannsen, 1905

Ablabesmyia longistyla Fittkau, 1962

LoCALIDADES: Río Lozoya, 30TVL292298; Río Jarama, 30TVL602214; Río Jarama, 30TVL565139 (Soriano, 2001). Canal de Carlos III (Las Rozas de Madrid), 30TVK248850. San Martín de la Vega, 30TVK5152

Ablabesmyia monilis (Linnaeus, 1758)

LOCALIDADES: Madrid (Czerny \& Strobl, 1909; Arias, 1912; Cobo et al., 1987; Soriano, 2001; Soriano et al. 1997).

Género CONCHAPELOPIA Fittkau, 1957

Conchapelopia pallidula (Meigen, 1818)

LoCALIDADES: Río Jarama, 30TVL565139 (Soriano, 2001).

Conchapelopia viator (Kieffer, 1911)

Localidades: Arroyo de la Poveda, 30TUK959948 (Soriano, 2001). 
Género LARSIA Fittkau, 1962

Larsia atrocincta (Goetghebuer, 1942)

LocAlidAdES: Río Lozoya, 30TVL292298; Arroyo de la Poveda, 30TUK959948 (Soriano et al., 1993; Soriano, 2001).

Género NILOTANYPUS Kieffer, 1923

\section{Nilotanypus dubius (Meigen, 1804)}

LOCALIDADES: Río Manzanares, 30TVL243109; Arroyo Navalahuerta, 30TVL264039; Río Lozoya, 30TVL292298; (Soriano, 2001).

Género PARAMERINA Fittkau, 1962

\section{Paramerina cingulata (Walker, 1856)}

LOCALIDADES: Río Cofio, 30TUK930866 (Soriano, 2001) Laguna del Garzo (Las Rozas de Madrid), 30TVK266889.

Paramerina divisa (Walker, 1856)

LOCALIDADES: Río Manzanares, 30TVL308057; Río Lozoya, 30TVL292298; Río Cofio, 30TUK882790; Río Perales, 30TVK847011; Río Jarama, 30TVL634239 (Soriano, 2001).

\section{Paramerina Pe 1 in Langton, 1984}

Localidades: Río Perales, 30TVK847011 (Soriano, 2001). Las Rozas de Madrid.

OBSERVACIONES: Según Langton (1984), este tipo de exuvia se corresponde problablente a una especie del género Paramerina. Sin embargo, podría ser incluida en el rango de variación de $P$. cingulata. Es necesario, por tanto, el análisis de más ejemplares para establecer su correcta identidad.

\section{Género RHEOPELOPIA Fittkau, 1962}

Rheopelopia maculipennis (Zetterstedt, 1838)

LOCALIDADES: Río Lozoya, 30TVL292298; Río Guadarrama, 30TVK168954 (Soriano, 2001).

\section{Género THIENEMANNIMYIA Fittkau, 1957}

Thienemannimyia carnea (Fabricius, 1805)

LocAlidAdes: Madrid (Czerny \& Strobl, 1909; Arias, 1912; Cobo et al., 1987; Soriano et al., 1997; Soriano, 2001).
Género TRISSOPELOPIA Kieffer, 1923

Trissopelopia longimana (Staeger, 1839)

LOCALIDADES: Río Aulencia, 30TVK158814; Río Guadalix, 30TVL333191; Arroyo de la Jarosa, 30TVL050034 (Soriano, 2001).

Subfamilia DIAMESINAE Kieffer, 1923

Tribu BOREOHEPTAGYIINI Brundin, 1966 Género BOREOHEPTAGYIA Brundin, 1966

Boreoheptagyia legeri (Goetghebuer, 1933)

LoCALIDADES: Río Guadalix, 30TVL333191; Arroyo de Canencia, 30TVL348268 (Soriano, 2001).

Tribu DIAMESINI Kieffer, 1923 Género DIAMESA Meigen, 1835

\section{Diamesa bertrami Edwards, 1935} 2001).

LoCALIDADES: Arroyo Peñalara, 30TVL196214 (Soriano,

Diamesa tonsa (Walker, 1856)

LOCALIDADES: Arroyo Peñalara, 30TVL196214 (Soriano et al.,1993; Soriano 1995).

\section{Género POTTHASTIA Kieffer, 1922}

Potthastia gaedii (Meigen, 1838)

LoCALIDADES: Río Lozoya, 30TVL292298; Río Lozoya, 30TVL354333; Río Lozoya, 30TVL606294; Río Jarama, 30TVL71945; Río Jarama, 30TVL634239; Río Jarama, 30TVL602214 (Soriano, 2001).

Género PSEUDODIAMESA Goetghebuer, 1939

Pseudodiamesa branickii (Nowicki, 1873) 2001).

LoCALIDADES: Arroyo Peñalara, 30TVL196214 (Soriano, Género SYMPOTTHASTIA Pagast, 1947

Sympotthastia spinifera Serra-Tosio, 1968

LOCALIDADES: Río Lozoya, 30TVL292298 (Soriano et al., 1993; Soriano, 2001).

\section{Sympotthastia zavreli Pagast, 1947}

LOCALIDADES: Río Guadalix, 30TVL333191 (Soriano, 2001). 
Subfamilia PRODIAMESINAE Saether, 1976 Género PRODIAMESA Kieffer, 1906

\section{Prodiamesa olivacea (Meigen, 1818)}

LocALIDADES: Río Lozoya, 30TVL292298; Arroyo de Canencia, 30TVL348268; Río Cofio, 30TUK930866; Río Guadalix, 30TVL33319; Río Guadarrama, 30TVK168954 (Soriano, 2001).

Subfamilia ORTHOCLADIINAE Kieffer, 1911 Género BRILLIA Kieffer, 1913

Brillia modesta (Meigen, 1830)

LoCALIDADES: Río Perales, 30TVK847011 (Soriano, 2001).

Género CARDIOCLADIUS Kieffer, 1912

Cardiocladius capucinus (Zetterstedt, 1850)

LocalidAdes: Río Lozoya, 30TVL354333; Arroyo de la Poveda, 30TUK959948; Río Cofio, 30TUK930866 (Soriano, 2001). San Fernando de Henares, 30TVK5476. San Martín de la Vega, 30TVK5152.

\section{Cardiocladius fuscus Kieffer, 1924}

LOCALIDADES: Río Lozoya, 30TVL354333; Río Lozoya, 30TVL606294; Arroyo de la Poveda, 30TUK959948; Río Alberche, 30TUK973632; Río Jarama, 30TVL565139 (Soriano, 2001). San Fernando de Henares, 30TVK5476.

\section{Género CORYNONEURA Winnertz, 1846}

\section{Corynoneura celtica Edwards, 1924}

LOCALIDADES: Río Lozoya, 30TVL292298; Arroyo Peñalara, 30TVL196214; Arroyo de la Poveda, 30TUK959948; Río Guadalix, 30TVL333191 (Soriano et al., 1993; Soriano, 2001).

Corynoneura coronata Edwards, 1924

LocalidAdes: Río Cofio, 30TUK930866 (Soriano et al., 1993; Soriano, 2001).

\section{Corynoneura edwardsi Brundin, 1949}

LOCALIDADES: Río Lozoya, 30TVL292298; Río Lozoya, 30TVL606294; Arroyo de la Poveda, 30TUK959948; Río Guadalix, 30TVL333191; Río Guadarrama, 30TVK198688 (Soriano, 2001).

Corynoneura lobata Edwards, 1924

LOCALIDADES: Río Aulencia, 30TVK066933; Río Jarama, 30TVL565139 (Soriano, 2001).

\section{Corynoneura scutellata Winnertz, 1846}

LoCALIDADES: Río Jarama, 30TVL602214 (Soriano, 2001). Laguna del Retamar (Las Rozas de Madrid) 30TVK205855.

\section{Corynoneura Pe 2a in Langton, 1991}

Localidades: Río Lozoya, 30TVL292298; Arroyo Peñalara, 30TVL196214; Arroyo de la Poveda, 30TUK959948 (Soriano, 2001).

Género CRICOTOPUS van der Wulp, 1874

Cricotopus (Cricotopus) albiforceps (Kieffer in Thienemann \& Kieffer, 1916)

LoCALIDADES: Río Lozoya, 30TVL292298; Río Lozoya, 30TVL354333; Río Lozoya, 30TVL606294; Arroyo de la Poveda, 30TUK959948; Río Jarama, 30TVL634239; Río Jarama, 30TVL602214; Río Jarama, 30TVL565139 (Soriano, 2001). San Fernando de Henares, 30TVK5476. San Martín de la Vega, 30TVK5152.

Cricotopus (Cricotopus) annulator Goetghebuer, 1927

LOCALIDADES: Río Aulencia, 30TVK158814; Río Jarama, 30TVL565139 (Soriano, 2001).

Cricotopus (Cricotopus) beckeri Hirvenoja, 1973

Localidades: Arroyo de la Poveda, 30TUK959948 (Soriano, 2001).

\section{Cricotopus (Cricotopus) bicinctus (Meigen, 1818)}

Localidades: Río Manzanares, 30TVL243109; Río Manzanares, 30TVL308057; Río Manzanares, 30TVL332872; Río Lozoya, 30TVL292298; Río Lozoya, 30TVL354333; Río Lozoya, 30TVL606294; Río Aulencia, 30TVK158814; Río Cofio, 30TUK882790; Río Perales, 30TVK025703; Río Guadarrama, 30TVK168954; Río Guadarrama, 30TVK820206; Río Jarama, 30TVL634239; Río Jarama, 30TVL602214; Río Jarama, 30TVL565139 (Soriano, 2001). San Fernando de Henares, 30TVK5476.

Cricotopus (Cricotopus) curtus Hirvenoja, 1973

LoCALIDADES: Río Jarama, 30TVL565139 (Soriano, 2001).

Cricotopus (Cricotopus) cylindraceus (Kieffer in Kieffer \& Thienemann, 1908)

LoCALIDADES: Río Lozoya, 30TVL354333; Río Lozoya, 30TVL606294; Río Guadarrama, 30TVK198688; Río Alberche, 30TUK973632; Río Jarama, 30TVL634239; Río Jarama, 30TVL602214 (Soriano, 2001). San Martín de la Vega, 30TVK5152.

Cricotopus (Cricotopus) festivellus (Kieffer, 1906) LOCALIDADES: San Fernando de Henares, 30TVK5476. 
Cricotopus (Cricotopus) fuscus (Kieffer, 1909)

LoCALIDADES: Río Lozoya, 30TVL354333 (Soriano, 2001).

Cricotopus (Cricotopus) pallidipes Edwards, 1929

LOCALIDADES: Río Lozoya, 30TVL292298 (Soriano, 2001).

Cricotopus (Cricotopus) similis Goetghebuer, 1921 2001).

LoCALIDADES: Río Guadarrama, 30TVK168954 (Soriano,

Cricotopus (Cricotopus) tibialis (Meigen, 1804)

Localidades: Río Lozoya, 30TVL354333; Río Lozoya, 30TVL606294 (Soriano, 2001).

Cricotopus (Cricotopus) tremulus (Linnaeus, 1758)

LoCALIDADES: Río Lozoya, 30TVL292298; Río Lozoya, 30TVL354333 (Soriano, 2001).

Cricotopus (Cricotopus) triannulatus (Macquart, 1826)

LoCALIDADES: Río Lozoya, 30TVL292298; Río Lozoya, 30TVL606294; Río Jarama, 30TVL634239; Río Jarama 30TVL565139 (Soriano, 2001). San Fernando de Henares, 30TVK5476.

Cricotopus (Cricotopus) trifasica Edwards, 1929

LoCALIDADES: Río Lozoya, 30TVL292298 (Soriano, 2001).

Cricotopus (Cricotopus) vierriensis Goetghebuer, 1935

Localidades: Río Manzanares, 30TVL243109; Río Lozoya, 30TVL292298; Río Lozoya, 30TVL354333; Río Lozoya, 30TVL606294; Río Aulencia, 30TVK158814; Río Jarama, 30TVL634239; Río Jarama, 30TVL602214; Río Jarama, 30TVL565139 (Soriano, 2001).

Cricotopus (Cricotopus) similis Langton, 1991

LocALIDADES: Río Lozoya, 30TVL292298 (Soriano, 2001).

Cricotopus (Isocladius) intersectus (Staeger, 1839)

LOCALIDADES: Laguna de San Juan (Titulcia), 30TVK550430.

Cricotopus (Isocladius) ornatus (Meigen, 1818)

LocalidADES: Madrid (Strobl, 1900, Cobo et al., 1987; Soriano, 2001). El Escorial (Czerny \& Strobl, 1909; Arias, 1912; Cobo et al., 1987; Soriano, 2001). San Fernando de Henares, 30TVK5476. San Martín de la Vega, 30TVK5152.

Cricotopus (Isocladius) speciosus Goetghebuer, 1921 2001).

LOCALIDADES: Arroyo de la Poveda, 30TUK959948 (Soriano,

Cricotopus (Isocladius) sylvestris (Fabricius, 1794)

LOCALIDADES: Río Lozoya, 30TVL292298; Río Lozoya, 30TVL354333; Río Aulencia, 30TVK066933; Río Cofio,
30TUK930866; Río Guadarrama, 30TVK168954; Río Guadarrama, 30TVK820206; Río Guadarrama, 30TVK198688; (¿Cobo et al., 1987?; Soriano, 2001). Laguna del Tío Soga (Las Rozas de Madrid), 30TVK269874. Laguna de San Juan, (Titulcia), 30TVK550430. San Fernando de Henares, 30TVK5476. San Martín de la Vega, 30TVK5152.

Cricotopus (Isocladius) tricinctus (Meigen, 1818)

LoCAlidAdes: Casa de campo (Strobl, 1900; Hirvenoja, 1973). Río Manzanares, 30TVL332872; Río Lozoya, 30TVL354333; Río Aulencia, 30TVK158814 (Soriano, 2001).

Cricotopus (Isocladius) trifasciatus (Meigen in Panzer, 1813)

LoCALIDADES: Madrid (Arias, 1912; Cobo et al., 1987; Soriano, 2001). Río Lozoya, 30TVL354333; Río Cofio, 30TUK882790; Río Guadarrama, 30TVK168954; Río Guadarrama, 30TVK820206; Río Alberche, 30TUK973632; Río Alberche, 30TUK973632 (Soriano, 2001). San Fernando de Henares, 30TVK5476. San Martín de la Vega, 30TVK5152.

Género EPOICOCLADIUS Sulc \& Zavrel, 1924

Epoicocladius flavens (Malloch, 1915)

LoCALIDADES: Río Manzanares, 30TVL243109 (Soriano, 2001).

Género EUKIEFFERIELLA Thienemann, 1926

Eukiefferiella brevicalcar (Kieffer, 1911)

Localidades: Río Guadalix, 30TVL333191; Arroyo Peñalara, 30TVL196214; Río Lozoya, 30TVL292298 (Soriano, 2001).

Eukiefferiella claripennis (Lundbeck, 1898)

LOCALIDADES: Arroyo Navacerrada, 30TVL160173; Río Manzanares, 30TVL308057; Arroyo Navalahuerta, 30TVL264039; Arroyo de la Poveda, 30TUK959948; Río Guadarrama, 30TVK168954; Río Guadarrama, 30TVK820206; Río Guadarrama, 30TVK198688; Río Alberche, 30TUK973632 (Soriano, 2001). Embalse de Molino de la Hoz (Las Rozas de Madrid), 30TVK205872. San Fernando de Henares, 30TVK5476. San Martín de la Vega, 30TVK5152.

Eukiefferiella clypeata (Kieffer, 1923)

LoCALIDADES: Río Lozoya, 30TVL292298; Arroyo de la Poveda, 30TUK959948; (Soriano, 2001).

Eukiefferiella coerulescens (Kieffer in Zavrel, 1926)

Localidades: Arroyo Peñalara, 30TVL196214; Río Lozoya, 30TVL292298; Arroyo de la Poveda, 30TUK959948; Río Jarama, 30TVL634239 (Soriano, 2001).

Eukiefferiella cyanea Thienemann, 1936

LOCALIDADES: Río Guadarrama, El Escorial y Madrid (Soriano, 2001). 
Eukiefferiella devonica (Edwards, 1929)

LOCALIDADES: Río Manzanares, 30TVL243109; Río Lozoya, 30TVL354333 (Soriano, 2001).

Eukiefferiella dittmari Lehmann, 1972

LocAlidAdES: Río Guadalix, 30TVL333191; Arroyo Peñalara, 30TVL196214 (Soriano et al., 1993; Soriano, 1995).

Eukiefferiella fuldensis Lehmann, 1972

LoCALIDADES: Río Manzanares, 30TVL243109; Arroyo Peñalara, 30TVL196214 (Soriano, 2001).

Eukiefferiella gracei (Edwards, 1929)

LOCALIDADES: Río Manzanares, 30TVL243109; Río Aulencia, 30TVK158814; Río Guadalix, 30TVL333191 (Soriano, 2001).

Eukiefferiella ilkleyensis (Edwards, 1929)

LOCALIDADES: Río Lozoya, 30TVL292298; Arroyo de la Poveda, 30TUK959948 (Soriano, 2001).

Eukiefferiella lobifera Goetghebuer, 1934

LOCALIDADES: Río Lozoya, 30TVL354333 (Soriano, 2001).

Eukiefferiella minor (Edwards, 1929)

LOCALIDADES: Río Lozoya, 30TVL292298 (Soriano, 2001).

Eukiefferiella tirolensis Goetghebuer, 1938

LOCALIDADES: Río Guadarrama, 30TVK168954; Arroyo Peñalara, 30TVL196214 (Soriano, 2001).

Género HELENIELLA Gouin, 1943

Heleniella ornaticollis (Edwards, 1929)

LOCALIDADES: Río Manzanares, 30TVL243109; Río Lozoya, 30TVL292298 (Soriano, 2001).

Género HETEROTRISSOCLADIUS Spärck, 1923

Heterotrissocladius marcidus (Walker, 1856

LoCALIDADES: Arroyo de Canencia, 30TVL348268; Río Jarama, 30TVL634239 (Soriano, 2001).

Género KRENOSMITTIA Thienemann \& Krüger, 1939

Krenosmittia boreoalpina (Goetghebuer, 1944)

LOCALIDADES: Arroyo de Canencia, 30TVL348268 (Soriano et al., 1993; Soriano, 2001).
Krenosmittia camptophlebs (Edwards, 1929)

LocAlidAdES: Río Guadarrama, El Escorial, Madrid (Wülker, 1957 a; Soriano, 2001). Río Jarama, 30TVL634239 (Soriano, 2001).

\section{Género LIMNOPHYES Eaton, 1875}

Lymnophyes edwarsi Saether, 1990

Localidades: Laguna del Tío Soga (Las Rozas de Madrid), 30TVK269874.

Limnophyes minimus (Meigen, 1818)

Localidades: Arroyo de la Poveda, 30TUK959948 (Soriano, 2001).

Limnophyes punctipennis (Goetghebuer, 1921)

LOCALIDADES: Río Cofio, 30TUK930866 (Soriano et al., 1993; Soriano, 2001).

Limnophyes Pe 1a in Langton, 1991

LoCALIDADES: Río Lozoya, 30TVL292298 (Soriano, 2001).

Género METRIOCNEMUS van der Wulp, 1874

Metriocnemus eurynotus (Holmgren, 1883)

LoCALIDADES: Río Manzanares, 30TVL308057; Río Guadarrama, 30TVK168954 (Soriano, 2001).

Género NANOCLADIUS Kieffer, 1913

Nanocladius bicolor (Zetterstedt, 1838)

LOCALIDADES: Río Aulencia, 30TVK158814; Río Guadarrama, 30TVK820206 Río Jarama, 30TVL602214; Río Alberche, 30TUK973632 (Soriano, 2001). San Fernando de Henares, 30TVK5476.

Nanocladius rectinervis (Kieffer, 1911)

LOCALIDADES: Río Manzanares, 30TVL243109; Arroyo Navalahuerta, 30TVL264039; Río Manzanares, 30TVL332872; Río Lozoya, 30TVL292298; Arroyo de la Poveda, 30TUK959948; Río Guadalix, 30TVL333191; Río Jarama, 30TVL602214; Río Jarama, 30TVL565139 (Soriano, 2001).

Género ORTHOCLADIUS van der Wulp, 1874

Orthocladius (Eudactylocladius) fuscimanus (Kieffer in Kieffer \& Thienemann, 1908)

LOCALIDADES: Río Lozoya, 30TVL354333 (Soriano, 2001). 
Orthocladius (Euorthocladius) ashei Soponis, 1990

LoCALIDADES: Río Lozoya, 30TVL292298; Río Lozoya, 30TVL354333; Arroyo de la Poveda, 30TUK959948; Río Guadalix, 30TVL333191; Río Jarama, 30TVL634239 (Soriano, 2001)

Orthocladius (Euorthocladius) rivicola Kieffer, 1921

LOCALIDADES: Río Lozoya, 30TVL354333; Río Guadalix, 30TVL333191 (Soriano, 2001).

Orthocladius (Euorthocladius) rivulorum Kieffer, 1909 LOCALIDADES: Río Lozoya, 30TVL292298 (Soriano, 2001).

Orthocladius (Euorthocladius) saxosus (Tokunaga, 1939)

LoCALIDADES: Río Lozoya, 30TVL292298 (Soriano, 2001).

Orthocladius (Euorthocladius) thienemanni Kieffer in Kieffer \& Thienemann, 1906

LOCALIDADES: Río Lozoya, 30TVL354333 (Soriano, 2001).

Orthocladius (Orthocladius) frigidus (Zetterstedt, 1838)

LoCALIDADES: Río Jarama, 30TVL602214 (Soriano, 2001).

Orthocladius (Orthocladius) obumbratus Johannsen, 1905

LOCALIDADES: Río Lozoya, 30TVL354333 (Soriano, 2001).

Orthocladius (Orthocladius) Pe 4 Langton, 1991

LOCALIDADES: Río Lozoya, 30TVL292298 (Soriano, 2001).

Género PARACLADIUS Hirvenoja, 1973

Paracladius alpicola (Zetterstedt, 1850)

LocAlidAdes: Madrid (Strobl, 1905). Río Jarama, 30TVL565139 (Soriano, 2001).

Paracladius conversus (Walker, 1856)

LOCALIDADES: Río Aulencia, 30TVK158814; Río Jarama, 30TVL634239 (Soriano, 2001).

Género PARACRICOTOPUS Thienemann \& Harnisch, 1932

\section{Paracricotopus niger (Kieffer, 1913)}

LocAlidAdES: Río Manzanares, 30TVL243109; Río Lozoya, 30TVL292298; Río Jarama, 30TVL602214 (Soriano, 2001).
Género PARAKIEFFERIELLA Thienemann, 1936

Parakiefferiella bathophila (Kieffer, 1912)

LocAlidAdES: Río Jarama, 30TVL602214; Arroyo Peñalara, 30TVL196214 (Soriano, 2001).

Parakiefferiella coronata (Edwards, 1929)

LOCALIDADES: Río Jarama, 30TVL634239 (Soriano, 2001).

Parakiefferiella wuelkeri Moubayed, 1994

LoCALIDADES: Madrid (Wülker, 1957a; Langton, 1994; Soriano, 2001).

Género PARAMETRIOCNEMUS Goetghebuer, 1932

Parametriocnemus stylatus (Kieffer, 1924)

LOCALIDADES: Río Manzanares, 30TVL308057; Río Lozoya, 30TVL292298; Río Lozoya, 30TVL354333; Arroyo de Canencia, 30TVL348268; Río Lozoya, 30TVL606294; Río Aulencia, 30TVK066933; Río Aulencia, 30TVK158814; Arroyo de la Poveda, 30TUK959948; Río Cofio, 30TUK930866; Río Cofio, 30TUK882790; Río Perales, 30TVK847011; Río Guadalix, 30TVL333191; Río Alberche, 30TUK973632; Río Jarama, 30TVL634239; Río Jarama, 30TVL602214; Río Jarama, 30TVL565139 (Soriano, 2001)

Género PARAPHAENOCLADIUS Thienemann, 1924

Paraphaenocladius impensus Walker, 1856

LocAlidades: Arroyo de la Poveda, 30TUK959948; Río Guadarrama, 30TVK198688; Río Jarama, 30TVL565139 (Soriano, 2001). Laguna del Retamar (Las Rozas de Madrid) 30TVK205855.

Género PARATRICHOCLADIUS Santos Abreu, 1918

Paratrichocladius rufiventris (Meigen, 1830)

LOCALIDADES: Río Manzanares, 30TVL317002; Río Lozoya, 30TVL292298; Río Lozoya, 30TVL354333; Río Lozoya, 30TVL606294; Río Aulencia, 30TVK066933; Río Aulencia, 30TVK158814; Arroyo de la Poveda, 30TUK959948; Río Cofio, 30TUK930866; Río Guadalix, 30TVL333191; Río Jarama, 30TVL602214; Río Jarama, 30TVL565139 (Soriano, 2001).

Paratrichocladius skirwithensis (Edwards, 1929)

Localidades: Arroyo de la Poveda, 30TUK959948 (Soriano, 2001).

Género PARATRISSOCLADIUS Zavrel, 1937

Paratrissocladius excerptus (Walker, 1856)

LoCALIDADES: Río Perales, 30TVK847011; Río Guadalix, 30TVL333191 (Soriano, 2001). 
Género PSECTROCLADIUS Kieffer, 1906

Psectrocladius (Allopsectrocladius) obvius (Walker, 1856)

LocAlidAdES: Lagunas de las Ceudas (Las Rozas de Madrid), 30TVK262871.

Psectrocladius (Psectrocladius) limbatellus (Holmgren, 1869)

LOCALIDADES: Laguna del Retamar (Las Rozas de Madrid), 30TVK205855. Lagunas de la Cervera (Las Rozas de Madrid), 30TVK223846. Lagunas de las Ceudas (Las Rozas de Madrid), 30TVK262871. San Fernando de Henares, 30TVK5476. San Martín de la Vega, 30TVK5152.

Psectrocladius (Psectrocladius) oxyura Langton, 1985

LOCALIDADES: Laguna del Retamar (Las Rozas de Madrid) 30TVK205855. Laguna del Garzo (Las Rozas de Madrid), 30TVK266889.

Psectrocladius (Psectrocladius) sordidellus (Zetterstedt, 1838)

LocAlidADES: Lagunas de la Cervera (Las Rozas de Madrid), 30TVK223846. Lagunas de las Ceudas (Las Rozas de Madrid), 30TVK262871.

Género PSEUDORTHOCLADIUS Goetghebuer, 1932

Pseudorthocladius filiformis (Kieffer in Kieffer \& Thienemann, 1908)

LOCALIDADES: Río Jarama, 30TVL634239 (Soriano et al., 1993; Soriano, 2001).

Género PSEUDOSMITTIA Goetghebuer, 1932

Pseudosmittia Pe 2 in Langton, 1991

LOCALIDADES: Río Cofio, 30TUK930866 (Soriano, 2001).

Género RHEOCRICOTOPUS Thienemann \& Harnish, 1932

Rheocricotopus (Psilocricotopus) atripes (Kieffer, 1913)

LoCALIDADES: Río Manzanares, 30TVL308057; Arroyo Navalahuerta, 30TVL264039; Río Manzanares, 30TVL317002; Río Manzanares, 30TVL332872; Río Lozoya, 30TVL292298; Río Lozoya, 30TVL354333; Arroyo de la Poveda, 30TUK959948; Río Cofio, 30TUK930866; Río Cofio, 30TUK882790; Río Guadarrama, 30TVK168954; Río Guadarrama, 30TVK820206; Río Guadarrama, 30TVK198688; Río Alberche, 30TUK973632; Río Jarama, 30TVL634239; Río Jarama, 30TVL565139; Río Aulencia, 30TVK158814 (Soriano, 2001). San Fernando de Henares, 30TVK5476. San Martín de la Vega, 30TVK5152.
Rheocricotopus (Psilocricotopus) chalybeatus (Edwards, 1929)

LOCALIDADES: San Fernando de Henares, 30TVK5476.

Rheocricotopus (Rheocricotopus) fuscipes (Kieffer, 1909)

LOCALIDADES: Arroyo Navacerrada, 30TVL160173; Río Manzanares, 30TVL308057; Río Guadalix, 30TVL333191; Arroyo Peñalara, 30TVL196214 (Soriano, 2001).

Género SMITTIA Holmgren, 1869

Smittia aterrima (Meigen, 1818)

LOCALIDADES: Madrid (Strobl, 1905).

Género SYNORTHOCLADIUS Thienemann, 1935

Synorthocladius semivirens (Kieffer, 1909)

LoCALIDADES: Río Lozoya, 30TVL292298; Río Lozoya, 30TVL354333; Río Jarama, 30TVL634239 (Soriano, 2001).

Género THIENEMANNIA Kieffer, 1909

Thienemannia gracilis Kieffer, 1909

LOCALIDADES: Arroyo de Canencia, 30TVL348268 (Soriano, 2001). Laguna del Tío Soga (Las Rozas de Madrid), 30TVK269874.

Género THIENEMANNIELLA Kieffer, 1911

Thienemanniella acuticornis (Kieffer, 1912)

LocAlidADES: Río Manzanares, 30TVL243109; Río Lozoya, 30TVL292298 (Soriano, 2001).

Thienemanniella clavicornis (Kieffer, 1911)

LOCALIDADES: Arroyo de la Poveda, 30TUK959948; Río Guadarrama, 30TVK168954 (Soriano, 2001).

Thienemanniella majuscula (Edwards, 1929) 2001).

LOCALIDADES: Arroyo de la Poveda, 30TUK959948 (Soriano,

Thienemanniella vittata (Edwards, 1924)?

LOCALIDADES: Arroyo de la Poveda, 30TUK959948; Río Guadarrama, 30TVK820206; Río Jarama, 30TVL565139 (Soriano, 2001).

OBSERVACIONES: La determinación es dudosa.

Thienemanniella Pe 2a in Langton, 1991

LOCALIDADES: Río Manzanares, 30TVL243109; Arroyo Navacerrada, 30TVL160173; Arroyo Navalahuerta, 
30TVL264039; Río Manzanares, 30TVL332872; Arroyo Peñalara, 30TVL196214; Río Lozoya, 30TVL292298; Río Lozoya, 30TVL354333; Arroyo de Canencia, 30TVL348268; Río Lozoya, 30TVL606294; Río Aulencia, 30TVK158814; Arroyo de la Poveda, 30TUK959948; Río Perales, 30TVK847011; Río Guadalix, 30TVL333191; Río Guadarrama, 30TVK168954; Río Guadarrama, 30TVK820206; Río Jarama, 30TVL634239; Río Jarama, 30TVL565139 (Soriano, 2001).

Thienemanniella Pe 2b in Langton, 1991

LOCALIDADES: Río Jarama, 30TVL565139 (Soriano, 2001).

Género TVETENIA Kieffer, 1922

Tvetenia bavarica (Goetghebuer, 1934)

LOCALIDADES: Río Lozoya, 30TVL292298; Río Lozoya, 30TVL354333; Río Cofio, 30TUK882790; Río Guadalix, 30TVL333191 (Soriano, 2001).

Tvetenia calvescens (Edwards, 1929)

LOCALIDADES: Río Manzanares, 30TVL243109; Río Lozoya, 30TVL292298; Arroyo de la Poveda, 30TUK959948; Río Guadalix, 30TVL333191 (Soriano, 2001). San Fernando de Henares, 30TVK5476.

\section{Tvetenia verralli (Edwards, 1929)}

LocAlidAdES: Río Manzanares, 30TVL243109; Río Lozoya, 30TVL292298; Río Lozoya, 30TVL354333; Arroyo de Canencia, 30TVL348268; Arroyo de la Poveda, 30TUK959948; Río Guadalix, 30TVL333191; Río Jarama, 30TVL602214 (Soriano, 2001).

Subfamilia CHIRONOMINAE Macquart, 1838 Tribu CHIRONOMINI Macquart, 1838 Género CHIRONOMUS Meigen, 1803

Chironomus (Chironomus) annularius Auctt., nec. De Geer, 1776 in Meigen, 1818

Localidades: Casa de Campo, Madrid (Strobl, 1900; Soriano, 2001).

OBSERVACIONES: La existencia de homonimias dificulta la signación de las citas a una especie concreta. Chironomus annularis De Geer, 1776 in Meigen, 1818; Chironomus annularis De Geer in Meigen, 1804 y las variantes annularia, annulatus y annhulatus pertenecen a Chironomus annulatus Autt. Chironomus annularius (De Geer, 1776) es sinonimo de Chironomus plumosus (Linnaeus, 1758) y, finalmente, Chironomus annularius Meigen, 1804 (homonimo secundario de Tipula annularis De Geer, 1776 al ser transferido a Chironomus) correponde a la especie Paratendipes albimanus (Meigen, 1818). Tan sólo la revisión del material de referencia podría aclarar la identidad específica de las citas.
Chironomus (Chironomus) dorsalis Auctt., nec Meigen, 1818

LoCALIDADES: Madrid (Strobl, 1905; Soriano, 2001). El Escorial (Czerny \& Strobl, 1909; Arias, 1912; Cobo et al.,1987; Soriano, 2001).

OBSERVACIONES: Einfeldia dorsalis (Meigen, 1818) fue descrita como Chironomus dorsalis Meigen, 1818 y es considerado actualmente como nomen dubium. Debido a la homonimia existente, las citas de Chironomus dorsalis aquí recogidas, y anteriores a 1924, podrían corresponder a Einfeldia dorsalis ya que no ha sido revisado el material al que se hace referencia.

Chironomus (Chironomus) luridus Strenzke, 1959

LocalidAdes: San Fernando de Henares, 30TVK5476.

Chironomus (Chironomus) nuditarsis (Linnaeus, 1758)

LoCALIDADES: San Martín de la Vega, 30TVK5152.

Chironomus (Chironomus) plumosus (Linnaeus, 1758)

LOCALIDADES: El Escorial, Madrid (Strobl, 1905; Czerny \& Strobl, 1909; Arias, 1912; Prat, 1980 a, b; Cobo et al., 1987; Soriano, 2001). En un "parque cercado de Madrid" (Strobl, 1905; Soriano, 2001). Lagunas de las Ceudas (Las Rozas de Madrid), 30TVK262871. San Fernando de Henares, 30TVK5476. San Martín de la Vega, 30TVK5152.

\section{Chironomus (Chironomus) prasinus Meigen, 1804}

LOCALIDADES: El Escorial, Madrid (Czerny \& Strobl, 1909; Arias, 1912; Soriano, 2001).

Chironomus (Chironomus) riparius Meigen, 1804

LOCALIDADES: El Escorial (Czerny \& Strobl, 1909; Arias, 1912; Cobo et al., 1987; Soriano, 2001). San Fernando de Henares, 30TVK5476. San Martín de la Vega, 30TVK5152.

OBSERVACIONES: Es necesario hacer constar que existen una gran cantidad de citas de Chironomus thummi o C. thummi thummi en trabajos generales, citas basadas en material larvario y cuya identificación es más que dudosa.

\section{Chironomus (Chironomus) venustus Staeger, 1839}

Localidades: Madrid (Strobl, 1905; Czerny \& Strobl, 1909; Soriano; 1995).

\section{Género CLADOPELMA Kieffer, 1921}

\section{Cladopelma virescens (Meigen, 1818)}

LOCALIDADES: El Escorial (Czerny \& Strobl; 1909; Arias, 1912; Cobo et al., 1987; Soriano, 2001). San Martín de la Vega, 30TVK5152. 
Género CRYPTOCHIRONOMUS Kieffer, 1918

Cryptochironomus rostratus Kieffer, 1921

LOCALIDADES: Río Jarama, 30TVL602214 (Soriano, 2001).

Cryptochironomus supplicans (Meigen, 1830)

LOCALIDADES: Río Jarama, 30TVL602214 (Soriano, 2001). San Martín de la Vega, 30TVK5152.

Género CRYPTOTENDIPES Lenz, 1941

Cryptotendipes usmaensis (Pagast, 1931)

LOCALIDADES: Río Jarama, 30TVL602214 (Soriano et al., 1993; Soriano, 2001).

Género DICROTENDIPES Kieffer, 1913

Dicrotendipes nervosus (Staeger, 1839)

LOCALIDADES: Madrid (Goetghebuer, 1931; ContrerasLichtenberg, 1986, Soriano, 2001).

Dicrotendipes peringueyanus (Kieffer, 1924)

LOCALIDADES: San Martín de la Vega, 30TVK5152.

Género ENDOCHIRONOMUS Kieffer, 1918

Endochironomus (Endochironomus) tendens (Fabricius, 1775)

LoCALIDADES: Madrid (¿Cobo et al., 1987?; Soriano, 2001).

OBSERVACIONES: La cita de Cobo et al. (1987) es dudosa, y en Soriano (1995) se recoge como tal.

Endochironomus (Endotendipes) dispar (Meigen, 1830)

LOCALIDADES: El Escorial, Madrid (Czerny \& Strobl, 1909; Arias, 1912; Cobo et al., 1987; Soriano; 1995).

\section{Género GLYPTOTENDIPES Kieffer, 1913}

\section{Glyptotendipes barbipes (Staeger, 1839)}

LOCALIDADES: Laguna de San Juan (Titulcia), 30TVK550430.

\section{Glyptotendipes gripekoveni (Kieffer, 1913)}

LOCALIDADES: Madrid (Michailova et al., 1994). San Fernando de Henares, 30TVK5476. San Martín de la Vega, 30TVK5152.

Glyptotendipes pallens (Meigen, 1804)

LOCALIDADES: Río Aulencia, 30TVK158814; Río Guadarrama, 30TVK168954; (Soriano, 2001). San Fernando de Henares, 30TVK5476. San Martín de la Vega, 30TVK5152.
Género HARNISCHIA Kieffer, 1921

Harnischia fuscimana Kieffer, 1921

LOCALIDADES: Río Jarama, 30TVL602214 (Soriano, 2001).

Género MICROTENDIPES Kieffer, 1915

Microtendipes britteni (Edwards, 1929)

LoCALIDADES: Río Lozoya, 30TVL354333 (Soriano, 2001).

Microtendipes chloris (Meigen, 1818)

LOCALIDADES: Arroyo de la Poveda, 30TUK959948; Río Cofio, 30TUK930866 (Soriano et al., 1993; Soriano, 2001). Laguna del Garzo (Las Rozas de Madrid), 30TVK266889. Canal de Carlos III (Las Rozas de Madrid), 30TVK248850.

Microtendipes diffinis (Edwards, 1929)

LOCALIDADES: Río Cofio, 30TUK930866 (Soriano, 2001).

Género PARACHIRONOMUS Lenz, 1921

Parachironomus arcuatus (Goetghebuer, 1919)

LOCALIDADES: Río Guadarrama, 30TVK168954; Río Guadarrama, 30TVK820206; Río Guadarrama, 30TVK198688 (Soriano, 2001). San Fernando de Henares, 30TVK5476. San Martín de la Vega, 30TVK5152.

Parachironomus Pe 2 in Langton, 1991

LOCALIDADES: Laguna de San Juan (Titulcia), 30TVK550430.

Parachironomus Pe 3 in Langton, 1991

LOCALIDADES: Río Lozoya, 30TVL292298; Río Guadarrama, 30TVK198688; Río Aulencia, 30TVK158814 (Soriano, 2001).

Género PARATENDIPES Kieffer, 1911

Paratendipes albimanus (Meigen, 1818)

LOCALIDADES: Madrid (Strobl, 1900; Arias, 1912; Cobo et al., 1987; Soriano et al., 1997; Soriano, 2001).

Paratendipes Pe 1 in Langton, 1984

Localidades: Río Cofio, 30TUK93086; Río Perales, 30TVK847011 (Soriano, 2001).

Género PHAENOPSECTRA Kieffer, 1921

Phaenopsectra flavipes (Meigen, 1818)

LOCALIDADES: Río Guadarrama, 30TVK198688 (Soriano, 2001). 
Género POLYPEDILUM Kieffer, 1912

Polypedilum (Pentapedilum) nubens (Edwards, 1929)

LOCALIDADES: Río Lozoya, 30TVL292298; Arroyo de la Poveda, 30TUK959948; Río Jarama, 30TVL634239; Río Jarama, 30TVL602214; Río Jarama, 30TVL565139 (Soriano, 2001).

Polypedilum (Pentapedilum) tritum (Walker, 1856)

LoCALIDADES: Río Jarama, 30TVL602214; (Soriano, 2001).

Polypedilum (Pentapedilum) uncinatum (Goetghebuer, 1921)

LOCALIDADES: Río Lozoya, 30TVL354333; Río Aulencia, 30TVK066933 Río Guadarrama, 30TVK820206; Río Jarama, 30TVL634239 (Soriano, 2001).

Polypedilum (Polypedilum) arundineti (Goetghebuer, 1921)

LocAlidADES: Laguna del Garzo (Las Rozas de Madrid), 30TVK266889.

Polypedilum (Polypedilum) convictum (Walker, 1856)

LOCALIDADES: Río Lozoya, 30TVL292298; Río Lozoya, 30TVL354333; Arroyo de la Poveda, 30TUK959948; Río Cofio, 30TUK930866; Río Cofio, 30TUK882790; Río Alberche, 30TUK973632; Río Jarama, 30TVL565139 (Soriano, 2001). Canal de Carlos III (Las Rozas de Madrid), 30TVK248850.

Polypedilum (Polypedilum) cultellatum Goetghebuer, 1931 LOCALIDADES: Río Lozoya, 30TVL606294 (Soriano, 2001).

Polypedilum (Polypedilum) laetum (Meigen, 1818)

LOCALIDADES: Río Jarama, 30TVL565139 (Soriano, 2001).

Polypedilum (Polypedilum) nubeculosum (Meigen, 1804)

LOCALIDADES: Río Alberche, 30TUK973632 (Soriano, 2001). Lagunas de las Ceudas (Las Rozas de Madrid), 30TVK262871.

Polypedilum (Polypedilum) pedestre (Meigen, 1830)

LOCALIDADES: Río Lozoya, 30TVL292298; Río Lozoya, 30TVL354333 (Soriano, 2001).

Polypedilum (Tripodura) bicrenatum Kieffer, 1921

LOCALIDADES: Río Jarama, 30TVL602214 (Soriano, 2001).

Polypedilum (Tripodura) nubifer (Skuse, 1889)

Localidades: Lagunas de las Ceudas (Las Rozas de Madrid), 30TVK262871. San Martín de la Vega, 30TVK5152.

Polypedilum (Tripodura) pullum (Zetterstedt, 1838)

Localidades: Río Manzanares; Arroyo de la Poveda, 30TUK959948; Río Aulencia, 30TVK158814 (Soriano, 2001). San Martín de la Vega, 30TVK5152.
Polypedilum (Tripodura) quadriguttatum Kieffer, 1921 1997).

LoCALIDADES: Madrid (Cobo et al., 1987; Soriano et al.,

Polypedilum (Tripodura) scalaenum (Schrank, 1803)

LOCALIDADES: El Escorial, Madrid (Czerny \& Strobl, 1909; Arias, 1912; Soriano, 2001). Río Guadarrama, 30TVK198688 (Soriano, 2001). San Martín de la Vega, 30TVK5152.

Polypedilum (Tripodura) tetracrenatum Hirvenoja, 1962

LOCALIDADES: Río Lozoya, 30TVL354333; Río Alberche, 30TUK973632; Río Jarama, 30TVL565139 (Soriano, 2001).

Género STENOCHIRONOMUS Kieffer, 1919

Stenochironomus gibbus (Fabricius, 1794)

LOCALIDADES: Río Jarama, 30TVL602214 (Soriano, 2001).

Género STICTOCHIRONOMUS Kieffer, 1919

Stictochironomus maculipennis (Meigen, 1818)

LoCALIDADES: Río Jarama, 30TVL602214 (Soriano, 2001).

Stictochironomus sticticus (Fabricius, 1794)

LoCALIDADES: Madrid (Strobl, 1900; Arias, 1912; Cobo et al., 1987; Soriano, 2001; Soriano et al., 1997). Río Jarama, 30TVL602214 (Soriano, 2001). San Fernando de Henares, 30 TVK5476.

Stictochironomus Pe 2 in Langton, 1991

Localidades: Arroyo de la Poveda, 30TUK959948 (Soriano, 2001)

Género XENOCHIRONOMUS Kieffer, 1921

Xenochironomus xenolabis (Kieffer in Thienemann \& Kieffer, 1916)

LOCALIDADES: Río Jarama, 30TVL602214 (Soriano, 2001).

Tribu TANYTARSINI Goetghebuer, 1937

Género CLADOTANYTARSUS Kieffer, 1921

Cladotanytarsus atridorsum Kieffer, 1924

LoCALIDADES: Río Jarama, 30TVL565139 (Soriano, 2001).

Cladotanytarsus mancus (Walker, 1856)

LoCALIDADES: Río Manzanares, Embalse de Santillana, 30TVL308057 Prat (1980a; Soriano, 2001). Río Manzanares, 
30TVL332872; Arroyo de la Poveda, 30TUK959948; Río Jarama, 30TVL634239; Río Jarama，30TVL602214; Río Jarama, 30TVL565139 (Soriano, 2001)

Cladotanytarsus vanderwulpi (Edwards, 1929)

LOCALIDADES: Río Jarama, 30TVL602214 (Soriano, 2001).

Cladotanytarsus Pe 5 a in Langton, 1991

LoCALIDADES: Río Lozoya, 30TVL354333 (Soriano, 2001).

Género MICROPSECTRA Kieffer, 1909

\section{Micropsectra atrofasciata (Kieffer, 1911)}

LoCALIDADES: Arroyo Navacerrada, 30TVL160173; Río Manzanares, 30TVL308057; Río Manzanares, 30TVL332872; Río Lozoya, 30TVL292298; Río Lozoya, 30TVL354333; Río Aulencia, 30TVK158814; Arroyo de la Poveda, 30TUK959948; Río Cofio, 30TUK930866; Río Cofio, 30TUK882790; Río Perales, 30TVK025703; Río Guadarrama, 30TVK168954; Río Guadarrama, 30TVK820206; Río Guadarrama, 30TVK198688; Río Alberche, 30TUK973632; Río Jarama, 30TVL602214; Río Jarama, 30TVL565139 (Soriano, 2001); Arroyo Peñalara, 30TVL196214.

Micropsectra bidentata (Goetghebuer, 1921)

LoCALIDADES: Río Guadalix, 30TVL333191 (Soriano, 2001)

Micropsectra contracta Reiss, 1965

LoCALIDADES: Río Guadalix, 30TVL333191 (Soriano, 2001). San Fernando de Henares, 30TVK5476. San Martín de la Vega, 30TVK5152.

Micropsectra junci (Meigen, 1818)

LoCALIDADES: El Escorial, Madrid (Czerny \& Strobl, 1909; Arias, 1912; Cobo et al., 1987; Soriano, 2001). Río Perales, 30TVK847011; Río Alberche, 30TUK973632 (Soriano, 2001).

\section{Micropsectra lindebergi Sawedal, 1976}

Localidades: Lagunas de las Ceudas (Las Rozas de Madrid), 30TVK262871.

Micropsectra lindrothi Goetghebuer in Goetghebuer \& Lindroth, 1931

Localidades: Arroyo de la Poveda, 30TUK959948 (Soriano, 2001). Lagunas de la Cervera (Las Rozas de Madrid), 30TVK223846. Lagunas de las Ceudas (Las Rozas de Madrid), 30TVK262871. Canal de Carlos III (Las Rozas de Madrid), 30TVK248850.

\section{Micropsectra notescens (Walker, 1856)}

Localidades: Río Lozoya, 30TVL292298 (Soriano, 2001).
Micropsectra radialis Goetghebuer, 1928

LOCALIDADES: San Fernando de Henares, 30TVK5476.

Género NEOZAVRELIA Goetghebuer, 1941

Neozavrelia Pe 1 in Langton, 1991

LOCALIDADES: Río Aulencia, 30TVK066933 (Soriano, 2001.)

Género PARATANYTARSUS Thienemann \& Bause, 1913

Paratanytarsus austriacus (Kieffer in Albrecht, 1924)

Localidades: Arroyo de la Poveda, 30TUK959948 (Soriano, 2001).

\section{Paratanytarsus dissimilis Johanssen, 1905}

LoCALIDADES: Río Manzanares, 30TVL243109; Río Manzanares, 30TVL332872; Río Lozoya, 30TVL292298; Río Lozoya, 30TVL3543333; Río Lozoya, 30TVL606294; Arroyo de la Poveda, 30TUK959948; Río Cofio, 30TUK930866; Río Cofio, 30TUK930866; Río Guadarrama, 30TVK820206; Río Guadarrama, 30TVK198688; Río Jarama, 30TVL634239; Río Jarama, 30TVL602214; Río Jarama, 30TVL565139 (Soriano, 2001). Laguna de San Juan (Titulcia), 30TVK550430.

Paratanytarsus grimmii (Schneider, 1885)

LOCALIDADES: Lagunas de las Ceudas (Las Rozas de Madrid), 30TVK262871. San Fernando de Henares, 30TVK5476. San Martín de la Vega, 30TVK5152.

Paratanytarsus inopertus (Walker, 1856)

LOCALIDADES: Arroyo de la Poveda, 30TUK959948; Río Guadarrama, 30TVK820206; Río Jarama, 30TVL565139 (Soriano, 2001).

Paratanytarsus natvigi (Goetghebuer, 1933)

LOCALIDADES: Río Jarama, 30TVL602214 (Soriano, 2001). Lagunas de las Ceudas (Las Rozas de Madrid), 30TVK262871. San Fernando de Henares, 30TVK5476.

Género RHEOTANYTARSUS Thienemann \& Bause, 1913

\section{Rheotanytarsus distinctissimus (Brundin, 1947)}

LoCALIDADES: Río Lozoya, 30TVL292298; Río Alberche, 30TUK973632; (Soriano, 2001).

Rheotanytarsus pentapoda (Kieffer, 1909)

LoCALIDADES: Río Manzanares, 30TVL308057; Río Lozoya, 30TVL354333; Río Lozoya, 30TVL606294; Arroyo de la Poveda, 30TUK959948; Río Cofio, 30TUK930866; Río 
Cofio, 30TUK882790; Río Guadalix, 30TVL333191; Río Jarama, 30TVL602214 (Soriano, 2001).

Rheotanytarsus photophilus (Goetghebuer, 1921)

LOCALIDADES: Río Jarama, 30TVL565139 (Soriano, 2001).

Rheotanytarsus reissi Lehmann, 1970

LocAlidADES: Río Manzanares, 30TVL243109; Río Lozoya, 30TVL354333; Río Guadalix, 30TVL333191; Río Jarama, 30TVL602214 (Soriano, 2001).

\section{Rheotanytarsus rhenanus Klink, 1983}

LOCALIDADES: Río Jarama, 30TVL634239; Río Jarama, 30TVL602214; Río Jarama, 30TVL565139 (Soriano et al., 1993; Soriano; 1995).

Género STEMPELLINA Thienemann \& Bause, 1913

Stempellina bausei (Kieffer, 1911)

LoCALIDADES: Río Lozoya, 30TVL292298; Río Jarama, 30TVL602214 (Soriano, 2001).

Género STEMPELLINELLA Brundin, 1947

Stempellinella brevis (Edwards, 1929) 2001).

LOCALIDADES: Río Manzanares, 30TVL243109 (Soriano,

Género TANYTARSUS van der Wulp, 1874

Tanytarsus brundini Lindeberg, 1963

LOCALIDADES: Río Manzanares, 30TVL243109; Río Lozoya, 30TVL292298; Río Lozoya, 30TVL606294; Río Aulencia, 30TVK066933; Río Perales, 30TVK847011; Río Jarama, 30TVL634239; Río Jarama, 30TVL602214 (Soriano, 2001).

Tanytarsus chinyensis Goetghebuer, 1934

LocAlidADES: Lagunas de las Ceudas (Las Rozas de Madrid), 30TVK262871.

Tanytarsus ejuncidus (Walker, 1856)

LOCALIDADES: Río Jarama, 30TVL602214 (Soriano, 2001).

Tanytarsus eminulus (Walker, 1856)

LOCALIDADES: Río Lozoya, 30TVL292298; Río Aulencia, 30TVK158814; Río Cofio, 30TUK930866; Río Alberche, 30TUK973632; Río Jarama, 30TVL565139 (Soriano, 2001).

Tanytarsus fimbriatus Reiss \& Fittkau, 1971

LOCALIDADES: San Fernando de Henares, 30TVK5476.
Tanytarsus gregarius Kieffer, 1909

LOCALIDADES: Embalse del Vellón, 30TVL425154 (Prat, 1980). Río Jarama, 30TVL565139 (Soriano, 2001)

Tanytarsus heusdensis Goetghebuer, 1923

LOCALIDADES: Río Lozoya, 30TVL292298; Río Jarama, 30TVL602214 (Soriano, 2001).

Tanytarsus medius Reiss \& Fittkau, 1971

LOCALIDADES: Río Aulencia, 30TVK158814; Río Jarama, 30TVL602214 (Soriano, 2001).

Tanytarsus mendax Kieffer, 1925 nec Reiss \& Fittkau, 1971

Localidades: Madrid (Cobo et al., 1987).

Tanytarsus pallidicornis (Walker, 1856)

LoCALIDADES: Río Lozoya, 30TVL292298; Río Jarama, 30TVL602214 (Soriano, 2001).

Tanytarsus quadridentatus Brundin, 1947

LoCALIDADES: Río Manzanares, 30TVL308057; Río Lozoya, 30TVL354333; Río Guadarrama, 30TVK820206; Río Jarama, 30TVL602214 (Soriano, 2001).

Tanytarsus striatulus Lindeberg, 1976

LOCALIDADES: Río Lozoya, 30TVL354333 (Soriano et al., 1993; Soriano, 2001).

Tanytarsus usmaensis Pagast, 1931

LoCALIDADES: Río Lozoya, 30TVL292298 (Soriano, 2001).

Género VIRGATANYTARSUS Pinder, 1982

Virgatanytarsus arduennensis (Goetghebuer, 1922)

LOCALIDADES: Río Manzanares, 30TVL332872; Río Lozoya, 30TVL354333; Río Lozoya, 30TVL606294; Río Lozoya, 30TVL606294; Río Cofio, 30TUK882790; Río Alberche, 30TUK973632; Río Jarama, 30TVL634239; Río Jarama, 30TVL602214; Río Jarama, 30TVL565139 (Soriano, 2001).

Virgatanytarsus triangularis (Goetghebuer, 1928)

LoCALIDADES: Río Manzanares, 30TVL332872; Río Lozoya, 30TVL354333; Río Lozoya, 30TVL606294; Río Cofio, 30TUK930866; Río Jarama, 30TVL565139 (Soriano, 2001).

\section{NOMINA DUBIA}

Chironomus flaveolus Meigen, 1818

LoCALIDADES: Madrid (Czerny \& Strobl, 1909; Arias, 1912). 
Tanytarsus latus (Staeger, 1839)

Chironomus latus Staeger, 1839

Chironomus (Tanytarsus) latus Meigen, 1818: Strobl (1905)

LoCALIDADES: Madrid: (Czerny \& Strobl, 1909; Arias 1912).

Tanytarsus pusio Meigen, 1830

Chironomus (Tanytarsus) pusio Meigen, 1830: Strobl $(1900,1905)$

LoCALIDADES: Madrid: (Czerny \& Strobl, 1909; Arias, 1912).

\section{Referencias}

ARIAS, J., 1912. Datos para el conocimiento de la distribución geográfica de los Dipteros de España. Memorias de la Real Sociedad Española de Historia Natural, VII: 61-246.

Cobo, F., Jiménez, M. T., CAsas, J. J., Vílchez, A., Soriano, O., Sevillano, M., Rieradevall, M., MuÑoz, I. \& Prat, N., 1987. Inventario faunístico de los quironómidos conocidos de la Península Ibérica. Alquibla, 12: 56-65.

Cobo, F., Soriano, O. \& BÁez, M., 2002. Chironomidae. In: Carles-Tolrá (Coord.). Catálogo de los Diptera de España, Portugal y Andorra. Monografias S.E.A., 8: $35-44$.

CONTRERAs-LiCHTENBERG, R., 1986. Beitrag zur kenntnis der weibchen in der westpäarktis verbreiteter arten des genus Dicrotendipes Kieffer, 1913 (Diptera, Nematocera, Chironomidae). Acta Biologica Debrecina. Supplementum Oecologica Hungarica, 2: 173-179.

Czerny, L. \& Strobl, G., 1909. Spanische Diptera III, Beitrag. Verhandlungen der kaiserlich-königlichen zoologisch-botanischen Gesellschaft in Wien, 59: 121-301.

EDWARDS, F. W., 1929. British non-biting midges (Diptera, Chironomidae). Transactions of the Royal Entomological Society of London, 77: 279-430.

Goetghebuer, M., 1931. Chironomides paléarctiques (Diptéres) conservés au Musée d'Histoire Naturelle de Vienne. Annalen des Naturhistorischen Museums in Wien, 46: 91-115.

Grodhaus, G., 1987. Endochironomus Kieffer, Tribelos Townes, Synendotendipes, n. gen., and Endotribelos, n. gen. (Diptera: Chironomidae) of the Nearctic Region. Journal of the Kansas Entomological Society, 60: 167-247.

HiRvenoja, M., 1973. Revision der gattung Cricotopus van der Wulp und ihrer Verwandten (Diptera, Chironomidae). Annales Zoologici Fennici, 10(1): 1-363.

Langton, P. H., 1984. A key to pupal exuviae of british Chironomidae. P. H. Langton. Cambridgeshire. 324 pp.

Langton, P. H., 1991. A Key to pupal exuviae of West Palaearctic Chironomidae. P. H. Langton. Cambridgeshire. $386 \mathrm{pp}$.
LANGTON, P. H. 1994. A redescription of Parakiefferiella sp. D. Wülker, the pupa of Parakiefferiella wülkeri Moubayed (Diptera: Chironomidae), a species new to Britain. British Journal of Entomology and Natural History, 7: 11-13.

Michailova, P., Rieradevall, M. \& Real, M., 1994. New chromosome arm combination of genus Glyptotendipes Kieff. (Diptera, Chironomidae). Caryologia, 47(1): 81-95.

PInDER, L. C. V., 1978. A key to the adult males of the British Chironomidae (Diptera) the non-biting midges. 2 volumes. Freshwater Biological Association Scientific Publications, 37. Vol. 1: 1-169 pp., Vol. 2: figs. 77-189.

Prat, N., 1980. Quironómidos de los embalses españoles (Diptera) (2a parte). Graellsia, 34: 59-119.

Rossaro, B., Casalegno, C. \& Lencioni, V., 2002. West Palaearctic species belonging to the subgenus Orthocladius s. str. (Diptera, Chironomidae: Orthocladiinae). Bollettino di Zoologia e di Bachicoltura, Serie II, 34: 227-232.

Rossaro, B., Lencioni, V. \& CAsalegno, C., 2003 [not "2002"]. Revision of West Palaearctic species of Orthocladius s. str. van der Wulp, 1874 (Diptera: Chironomidae: Orthocladiinae), with a new key to species. Studi Trentini di Scienze Naturali - Acta Biologica, 79: 213-241.

SÆTHER, O. A., 2004. The chironomids (Diptera, Chironomidae) described by Lundström (1915) from arctic Siberia,with a redescription of Derotanypus sibiricus (Kruglova \& Chernovskii). Zootaxa, 595: 1-35.

Soriano, O., 2001. Los Quironómidos (Diptera, Chironomidae) de Madrid. Efecto de la regulación ejercida por el embalse del Vado (Guadalajara, España) sobre una comunidad de Quironómidos. Tesis doctoral, Universidad Complutense (Madrid). CD-Rom.

SorianO, O., RAmiL, J. \& CoBO, F., 1993. Adiciones a la fauna ibérica de quironómidos (Diptera: Chironomidae). Graellsia, 49: 97-103.

Soriano, O., Cobo, F., Rieradevall, M. \& Prat, N., 1997. Lista faunística y bibliográfica de los quironómidos (Diptera, Chironomidae) de la península Ibérica e islas Baleares. Listas de la flora y fauna de las aguas continentales de la península Ibérica, Asociación Española de Limnología. Madrid. 210 pp

Strobl, G., 1900. Spanische Dipteren (Chironomidae). Wiener Entomologische Zeitung, 19(VI y VII): 169-174.

Strobl, G., 1905. Spanischen Dipteren (Chironomidae). Memorias de la Real Sociedad Española de Historia Natural, 3: 396-404.

WülKER, V. W., 1957. Über die chironomiden der Parakiefferiella-Gruppe (Diptera: Tendipedidae, Orthocladiinae). Beiträge zur Entomologie, 7(3/4): 411-429. 